TRANSACTIONS OF THE

AMERICAN MATHEMATICAL SOCIETY

Volume 355, Number 12, Pages 4881-4900

S 0002-9947(03)03236-7

Article electronically published on July 28, 2003

\title{
STRATIFIED TRANSVERSALITY BY ISOTOPY
}

\author{
C. MURolo, D. J. A. TROtMAn, AND A. A. DU Plessis
}

\begin{abstract}
For $\mathcal{X}$ an abstract stratified set or a $(w)$-regular stratification, hence for any $(b)$-, $(c)$ - or $(L)$-regular stratification, we prove that after stratified isotopy of $\mathcal{X}$, a stratified subspace $\mathcal{W}$ of $\mathcal{X}$, or a stratified map $h: \mathcal{Z} \rightarrow \mathcal{X}$, can be made transverse to a fixed stratified map $g: \mathcal{Y} \rightarrow \mathcal{X}$.
\end{abstract}

\section{INTRODUCTION}

Let $\mathcal{X}=(A, \Sigma)$ be a stratified space (see $\S 2$ for the definition). A substratified object of $\mathcal{X}$ is a stratified space $\mathcal{W}=\left(W, \Sigma_{\mathcal{W}}\right)$, where $W$ is a subset of $A$, such that each stratum in $\Sigma_{\mathcal{W}}$ is contained in a single stratum of $\mathcal{X}$.

In this paper we consider the problem of putting a substratified object $\mathcal{W}$ of $\mathcal{X}$ in transverse position with respect to a fixed substratified object $\mathcal{V}$ of $\mathcal{X}$ or more generally with respect to a fixed stratified map $g: \mathcal{Y} \rightarrow \mathcal{X}$, for some stratified space $\mathcal{Y}$. More precisely, we deform $\mathcal{W}$ via a stratified isotopy $\Phi: \mathcal{X} \times I \rightarrow \mathcal{X}$ to a substratified object $\mathcal{W}^{\prime}=\Phi_{1}(\mathcal{W})$ of $\mathcal{X}$ which is transverse to $\mathcal{V}$ in $\mathcal{X}$, or more generally transverse to $g$.

This problem was resolved by Clint McCrory for stratified polyhedra 20, 21; his result is essential to the foundations of intersection homology [13. For Whitney (b)-regular stratifications Mark Goresky stated, with only a sketch of a proof, such a transversality theorem $((5.3)$ in [12]), valid for a restricted class of substratified objects, and only for controlled maps $g: \mathcal{Y} \rightarrow \mathcal{X}$. This result is essential in proving his main theorems about representing homology and cohomology by stratified objects $(3.4,4.7$ and 6.2 of [12]). In this paper we adapt the proof indicated in [12, to give a version with less restrictive hypotheses which allows one to develop further Goresky's geometric homology theory [24]. Some details are given at the end of this introduction.

Goresky's result applies to those substratified objects $\mathcal{W}$ of $\mathcal{X}$ satisfying a $\pi$-fibre condition with respect to a fixed system of control data $\mathcal{F}=\left\{\left(\pi_{X}, \rho_{X}\right): T_{X} \rightarrow\right.$ $X \times[0, \infty)\}_{X \in \Sigma}$, and to a stratified map $g: \mathcal{Y} \rightarrow \mathcal{X}$ which is controlled with respect to two systems of control data. The $\pi$-fibre condition says that $W$ is locally, near each point $x$ of $A$, a union of fibres of the projection $\pi_{S}: T_{S} \rightarrow S$, where $S$ is the stratum containing $x$, while the control condition on the map $g$ imposes a similar property for the fibres of $g$ (and of $\pi_{S}$ ). These conditions were used by Goresky to preserve transversality with respect to $g$ of a deformation $\mathcal{W}^{\prime}$ of $\mathcal{W}$ in his inductive proof.

Received by the editors October 2, 2001 and, in revised form, June 4, 2002.

2000 Mathematics Subject Classification. Primary 58A35, 57N75; Secondary 57N80, 57R52.

The first author received support from the Department of Mathematics of the Faculty of Engineering and the Office of International Relations of the University of Naples. 
Goresky's transversality theorem has been shown to be very useful in several important applications [12], [22], 223]; but the hypotheses of $\pi$-fibre on $\mathcal{W}$ and control on $g$ prevent a wider use.

The stratified transversality theorem we give in this paper holds for the most important types of regular stratifications, and for every stratified map without assuming control conditions. In particular, we obtain an analogue of Goresky's theorem for stratified maps $g: \mathcal{Y} \rightarrow \mathcal{X}$ which are not necessarily controlled and for substratified objects $\mathcal{W}$ which are not necessarily $\pi$-fibre. We also obtain the analogous theorem for two arbitrary stratified maps.

We acknowledge with thanks the referee's detailed comments on an earlier version of this work.

The contents of the paper are as follows.

In $\S 2$ we review the most important classes of regular stratifications: the abstract stratified sets of Thom and Mather 32], [18, [19], Whitney (b)-regular stratifications [36], the (c)-regular stratifications of Karim Bekka [3], the (w)-regular stratifications of Verdier 34] and the $(L)$-regular stratifications of Mostowski and Parusiński [29]. We briefly recall the relations between them.

In $\S 3$ we prove a transversality theorem (Theorem 3.8) for an abstract stratified set $\mathcal{X}$, thus including stratifications which are $(c)$-regular [3] or $(b)$-regular [18, [19]. This shows that, given a substratified object $\mathcal{V}$ of $\mathcal{X}$, every other substratified object $\mathcal{W}$ of $\mathcal{X}$ may be deformed by a stratified isotopy $\Phi: \mathcal{X} \times I \rightarrow \mathcal{X}$ to a substratified object $\mathcal{W}^{\prime}=\Phi_{1}(\mathcal{W})$ of $\mathcal{X}$ which is transverse to $\mathcal{V}$ in $\mathcal{X}$. Moreover, $\mathcal{W}^{\prime}$ may be chosen in an arbitrarily small neighbourhood of $\mathcal{W}$. Then we give a relative version (3.9), and show that similar proofs apply to $(w)$-regular stratifications (Theorem $3.13)$.

One can ask whether in the stratified transversality theorem one can require that $\mathcal{V} \cap \mathcal{W}^{\prime}$ and $\mathcal{V} \cup \mathcal{W}^{\prime}$ be again stratified spaces. We conclude the section by giving examples showing that this is not always so. Our result is thus the best possible in this category. On the other hand, cases when this can be done have important applications.

In $\S 4$ we improve Theorems 3.8, 3.9 and 3.13 by showing that for stratified maps $g: \mathcal{Y} \rightarrow \mathcal{X}$ and $h: \mathcal{Z} \rightarrow \mathcal{X}$ there exists a deformation by isotopy $h^{\prime}$ of $h$ in $\mathcal{X}$ which is transverse to $g$ (Theorem 4.4). The proof, by induction on the dimension $k$ of a skeleton $\mathcal{X}_{k}$ of $\mathcal{X}$, applies to stratifications for which good lifting of vector fields exists, in particular to abstract stratified sets and to $(w)$-regular stratifications (and hence to $(b)-,(c)$ - and $(L)$-regular stratifications).

If $h: \mathcal{W} \hookrightarrow \mathcal{X}$ is a stratified inclusion (i.e. $\mathcal{W}$ is the substratified object of $\mathcal{X}$ to deform), we seek a substratified object $\mathcal{W}^{\prime}$, isotopic to $\mathcal{W}$ and transverse to $g$. The transversalising deformation $\Phi_{1}: \mathcal{X} \rightarrow \mathcal{X}$ such that $\mathcal{W}^{\prime}=\Phi_{1}(\mathcal{W})$ is the time 1 homeomorphism of a stratified isotopy $\Phi=\left\{\Phi_{t}=\Phi_{t}^{n} \circ \cdots \circ \Phi_{t}^{1}: \mathcal{X} \rightarrow \mathcal{X}\right\}_{t \in I}$, obtained by composition of one-parameter groups $\left\{\Phi_{t}^{i}: \mathcal{X} \rightarrow \mathcal{X}\right\}_{t \in \mathbb{R}}$, which are the flows of continuous liftings of vector fields [26].

In $\S 5$ we consider the problem of preserving regularity conditions on substratified objects $\mathcal{W}$ after deformation (that is, the problem of the regularity of $\mathcal{W}^{\prime}$ ). The possibility of lifting vector fields continuously is useful here; also the notion of semidifferentiable stratified map introduced in [24]; see [25]. When $\mathcal{X}$ is $(c)$-regular and the time 1 map of the transversalising isotopy $\Phi_{1}: A \rightarrow A$ is semi-differentiable, 
then $\mathcal{W}^{\prime}=\Phi_{1}(\mathcal{W})$ is $(a)$ - or $(c)$-regular if $\mathcal{W}$ is respectively $(a)$ - or $(c)$-regular (Proposition 5.2).

When $\mathcal{X}$ has a Whitney $(b)$-regular stratification it is unknown whether semidifferentiability of $\Phi_{1}: \mathcal{X} \rightarrow \mathcal{X}$ suffices to preserve $(b)$-regularity. Such preservation would be useful for Goresky's "Whitney homology theory" $\left\{W H_{*}, W H^{*}\right\}$ ([11, [12, [22], 23]), providing an approach to a celebrated conjecture: "The representation map $R: W H_{k}(\mathcal{X}) \rightarrow H_{k}(\mathcal{X})$ is a bijection for every Whitney stratification $\mathcal{X}$ " [1], [12. This was the original motivation of our work. The first author has used the results of the present paper to extend Goresky's theory of homology and cohomology to abstract stratified sets and $(c)$-regular stratifications, so obtaining new homology theories $\left\{A H_{*}, A H^{*}\right\}$ and $\left\{B H_{*}, B H^{*}\right\}([24$, chapter IV, and [5]).

In these stratified homology theories, the transversality theorem proved here allows one to give geometric meaning to the operations of sum, cup, cap and cross product, analogous to the results of [11, [12, 22], 23]. It also gives geometrical sense to induced homomorphisms and to the Poincaré duality homomorphism.

The transversality theorem thus plays a similar role to the "Moving Lemma" in the theory of Chow rings $\mathrm{CH}_{*}$ for algebraic cycles of an algebraic variety [7].

\section{REgular STRATIFIED SPACES}

We recall that a stratification of a topological space $A$ is a locally finite partition $\Sigma$ of $A$ into $C^{1}$ connected manifolds (called the strata of $\Sigma$ ) satisfying the frontier condition: if $X$ and $Y$ are disjoint strata such that $X$ intersects the closure of $Y$, then $X$ is contained in the closure of $Y$. We then write $X<Y$ and $\partial Y=\bar{Y}-Y$, so that $\bar{Y}=Y \sqcup\left(\bigsqcup_{X<Y} X\right)$ and $\partial Y=\bigsqcup_{X<Y} X \quad(\sqcup=$ disjoint union $)$.

The pair $\mathcal{X}=(A, \Sigma)$ is called a stratified space with support $A$ and stratification $\Sigma$. The union of the strata of dimension $\leq k$ is called the $k$-skeleton, denoted by $A_{k}$, inducing a stratified space $\mathcal{X}_{k}=\left(A_{k}, \Sigma_{\mid A_{k}}\right)$.

A stratified map $f: \mathcal{X} \rightarrow \mathcal{X}^{\prime}$ between stratified spaces $\mathcal{X}=(A, \Sigma)$ and $\mathcal{X}^{\prime}=$ $\left(B, \Sigma^{\prime}\right)$ is a continuous map $f: A \rightarrow B$ which sends each stratum $X$ of $\mathcal{X}$ into a unique stratum $X^{\prime}$ of $\mathcal{X}^{\prime}$, such that the restriction $f_{X}: X \rightarrow X^{\prime}$ is smooth. We call such a map $f$ a stratified homeomorphism if $f$ is a global homeomorphism and each $f_{X}$ is a diffeomorphism.

A stratified vector field on $\mathcal{X}$ is a family $\zeta=\left\{\zeta_{X}\right\}_{X \in \Sigma}$ of vector fields such that $\zeta_{X}$ is a smooth vector field on the stratum $X$.

Extra conditions may be imposed on the stratification $\Sigma$, such as to be an abstract stratified set in the sense of Thom-Mather ([8], [18], 29], [35]) or, when $A$ is a subset of a $C^{1}$ manifold, to satisfy conditions $(a)$ or $(b)$ of Whitney ([18, [19, [36]), or $(c)$ of K. Bekka ([2], [3]) or, when $A$ is a subset of a $C^{2}$ manifold, to satisfy conditions $(w)$ of Kuo and Verdier [34], or $(L)$ of Mostowski (see [29]).

Now we recall these conditions and their more important relations.

Definition 2.1 (Thom and Mather). Let $\mathcal{X}=(A, \Sigma)$ be a stratified space.

A family $\mathcal{F}=\left\{\left(\pi_{X}, \rho_{X}, T_{X}\right)\right\}_{X \in \Sigma}$ is called a system of control data for $\mathcal{X}$ if for each stratum $X$ we have that:

1) $T_{X}$ is a neighbourhood of $X$ in $A$ (called a tubular neighbourhood of $X$ );

2) $\pi_{X}: T_{X} \rightarrow X$ is a continuous retraction of $T_{X}$ onto $X$ (called projection on $X)$;

3) $\rho_{X}: T_{X} \rightarrow[0, \infty)$ is a continuous function such that $X=\rho_{X}^{-1}(0)$ (called the distance function from $X$ ) 
and, furthermore, for every pair of adjacent strata $X<Y$, by considering the restriction maps $\pi_{X Y}=\pi_{X \mid T_{X Y}}$ and $\rho_{X Y}=\rho_{X \mid T_{X Y}}$, on the subset $T_{X Y}=T_{X} \cap Y$, we have that:

$5)$ the map $\left(\pi_{X Y}, \rho_{X Y}\right): T_{X Y} \rightarrow X \times(0, \infty)$ is a smooth submersion (it follows in particular that $\operatorname{dim} X<\operatorname{dim} Y)$;

6) for every stratum $Z$ of $\mathcal{X}$ such that $Z>Y>X$ and for every $z \in T_{Y Z} \cap T_{X Z}$ the following control conditions are satisfied:

i) $\pi_{X Y} \pi_{Y Z}(z)=\pi_{X Z}(z)$ (called the $\pi$-control condition), and

ii) $\rho_{X Y} \pi_{Y Z}(z)=\rho_{X Z}(z)$ (called the $\rho$-control condition).

In what follows we will set $T_{X}(\epsilon)=\rho_{X}^{-1}([0, \epsilon)), \forall \epsilon \geq 0$, and without loss of generality will assume $T_{X}=T_{X}(1)[8]$, [19].

If $A$ is Hausdorff, locally compact and admits a countable basis for its topology, the pair $(\mathcal{X}, \mathcal{F})$ is called an abstract stratified set. Since one usually works with a unique system of control data of $\mathcal{X}$, in what follows we will omit $\mathcal{F}$.

If $\mathcal{X}$ is an abstract stratified set, then $A$ is metrizable and the tubular neighbourhoods $\left\{T_{X}\right\}_{X \in \Sigma}$ may (and will always) be chosen such that: " $T_{X Y} \neq \emptyset$ if and only if $X<Y$ or $X>Y$ or $X=Y$ " (see [19, page 41 and following).

Let $f: \mathcal{X} \rightarrow \mathcal{X}^{\prime}$ be a stratified map between two abstract stratified sets, and let us fix two systems of control data

$$
\mathcal{F}=\left\{\left(T_{X}, \pi_{X}, \rho_{X}\right)\right\}_{X \in \Sigma} \quad \text { and } \quad \mathcal{F}^{\prime}=\left\{\left(T_{X^{\prime}}, \pi_{X^{\prime}}, \rho_{X^{\prime}}\right)\right\}_{X^{\prime} \in \Sigma^{\prime}}
$$

respectively of $\mathcal{X}$ and $\mathcal{X}^{\prime}$. The map $f$ is called controlled (with respect to $\mathcal{F}$ and $\left.\mathcal{F}^{\prime}\right)$ if when $X<Y$ there exists $\epsilon>0$ such that for all $y \in T_{X Y}(\epsilon)=T_{X}(\epsilon) \cap Y$ the following two control conditions hold:

$$
\begin{cases}\pi_{X^{\prime} Y^{\prime}} f_{Y}(y)=f_{X} \pi_{X Y}(y) & (\text { the } \pi \text {-control condition for } f), \\ \rho_{X^{\prime} Y^{\prime}} f_{Y}(y)=\rho_{X Y}(y) & (\text { the } \rho \text {-control condition for } f) .\end{cases}
$$

Similarly, a stratified vector field $\zeta=\left\{\zeta_{X}\right\}_{X \in \Sigma}$ is controlled (with respect to $\mathcal{F}$ ) if the following two control conditions hold:

$$
\begin{cases}\pi_{X Y *}\left(\zeta_{Y}(y)\right)=\zeta_{X}\left(\pi_{X Y}(y)\right) & (\text { the } \pi \text {-control condition for } \zeta) \\ \rho_{X Y *}\left(\zeta_{Y}(y)\right)=0 & (\text { the } \rho \text {-control condition for } \zeta)\end{cases}
$$

The notion of system of control data of $\mathcal{X}$, introduced by Mather in 19, is the fundamental tool allowing one to obtain good extensions of vector fields. In fact, we have [8], [19]

Proposition 2.2. If $\mathcal{X}$ is an abstract stratified set, every vector field $\zeta_{X}$ defined on a stratum $X$ of $\mathcal{X}$ admits a stratified $(\pi, \rho)$-controlled lifting $\zeta_{T_{X}}=\left\{\zeta_{Y}\right\}_{Y \geq X}$ defined on a tubular neighbourhood $T_{X}$ of $X$. Moreover, if $\zeta_{X}$ admits a global flow $\left\{\phi_{t}: X \rightarrow X\right\}_{t \in \mathbb{R}}$, then such a lifting $\zeta_{T_{X}}$ admits again a global flow $\left\{\phi_{T_{X} t}: T_{X} \rightarrow\right.$ $\left.T_{X}\right\}_{t \in \mathbb{R}}$, and every $\phi_{T_{X} t}: T_{X} \rightarrow T_{X}$ is a stratified, continuous and $(\pi, \rho)$-controlled homeomorphism.

Definition 2.3 (K. Bekka 1990). A stratified space $(A, \Sigma)$ is called (c)-regular if, for every stratum $X \in \Sigma$, there exist an open neighbourhood $U_{X}$ of $X$ in $\mathbb{R}^{n}$ and a $C^{1}$ function $\rho_{X}: U_{X} \rightarrow[0, \infty)$ such that $\rho_{X}^{-1}(0)=X$, and such that its stratified restriction to the star of $\mathcal{X}$,

$$
\rho_{X}: \operatorname{Star}(X) \cap U_{X} \rightarrow[0, \infty) \quad \text { is a Thom map, }
$$


where $\operatorname{Star}(X)=\bigcup_{Y \in \Sigma, Y \geq X} Y$ and the stratification on $\operatorname{Star}(X) \cap U_{X}$ is induced by $\Sigma$.

In substance, the $(c)$-regularity of Bekka means that for every pair of adjacent strata $X<Y$, the tangent spaces at $y \in Y$ to the level hypersurfaces $\rho_{X}^{-1}(\epsilon)$ (where $\left.\epsilon=\rho_{X}(y)\right)$ have limits which contain $T_{x} X$ when $y \rightarrow x \in X$.

Definition 2.4 (Verdier 1976). For any vector subspaces $U, V$ of $\mathbb{R}^{n}$, denote

$$
\delta(U, V)=\sup _{u \in U,\|u\|=1} \inf _{v \in V}\|u-v\| .
$$

A stratified space $(A, \Sigma)$ with $A \subseteq \mathbb{R}^{n}$ is called $(w)$-regular if, for every pair of strata $X<Y \in \Sigma$ and every $x_{0} \in X$ there exist a neighbourhood $U_{x_{0}}$ of $x_{0}$ on $\mathbb{R}^{n}$ and a constant $C_{x_{0}}>0$ such that

$$
\delta\left(T_{y} Y, T_{x} X\right) \leq C_{x_{0}}\|y-x\| \quad \forall x \in U_{x_{0}} \cap X \quad \text { and } \quad \forall y \in U_{x_{0}} \cap Y .
$$

We recall now the most important properties of lifting of vector fields on such regular stratifications and the most useful relations between them.

i) The condition "to be a Thom-Mather abstract stratified set" implies the existence of controlled lifting of vector fields [19].

ii) Bekka's (c)-regularity is characterized by the existence of continuous and controlled lifting of vector fields 30, and implies the condition "to be a ThomMather abstract stratified set" [2, 3]. Moreover for (c)-regular stratifications one can also find systems of control data whose maps $\left\{\left(\pi_{X}, \rho_{X}\right): T_{X} \rightarrow X \times[0, \infty)\right\}_{X}$ are $C^{1}[2,3]$.

iii) (b)-regularity implies (c)-regularity [2], [3].

$i v$ ) Verdier's $(w)$-regularity is characterized by the existence of rugose lifting of vector fields [6], [34].

$v)(L)$-regularity is characterized by the existence of Lipschitz lifting of vector fields 29 and implies $(w)$-regularity.

Finally, recall the following important facts.

A) Every abstract stratified set admits a (b)-regular embedding [11, [27, [31, and even [28] a subanalytic $(w)$-regular (and hence $(b)$-regular [15, 34]) embedding in some $\mathbb{R}^{N}$.

$B$ ) The first isotopy theorem of Thom holds for all the kinds of stratification considered above, using the (claimed) properties of stratified lifting of vector fields.

\section{Stratified ISOTOPY AND TRANSVERSALity OF SUBSTRATIFIED OBJECTS}

In analogy with the definition of a Whitney substratified object $\mathcal{V}$ of $\mathcal{X}$ given by Goresky in [12] for $\mathcal{V}$ and $\mathcal{X}$ which are Whitney stratified ((b)-regular), we give the following definition, where $(E)$ means one of the regularity conditions: "to be an abstract stratified set", the condition (b) of Whitney, the condition (c) of Bekka, $(w)$ of Verdier, or the condition $(L)$ of Mostowski.

Definition 3.1. Let $\mathcal{X}=(A, \Sigma)$ be a stratified space. An $(E)$-regular substratified object of $\mathcal{X}$ is a stratified space $\mathcal{V}=\left(V, \Sigma_{\mathcal{V}}\right)$, such that $V$ is a closed subset of $A, \Sigma_{\mathcal{V}}$ is $(E)$-regular and each stratum of $\mathcal{V}$ is contained in a unique stratum of $\mathcal{X}$. If $\mathcal{X}$ and $\mathcal{V}$ are both abstract stratified sets, then we say that $\mathcal{V}$ is an abstract substratified set of $\mathcal{X}$.

If $\mathcal{V}$ is a substratified object of $\mathcal{X}$, then the inclusion $i: \mathcal{V} \hookrightarrow \mathcal{X}$ is a stratified map and for each stratum $S$ of $\mathcal{X}, V \cap S$ is a closed subset of $S$ with an induced 
stratification $\mathcal{V}_{S}=\{R$ stratum of $\mathcal{V} \mid R \subseteq S\}$ : the restriction of $\mathcal{V}$ to the stratum $S$ of $\mathcal{X}$.

In what follows, when this is not ambiguous, we write simply $V$ for a substratified object $\mathcal{V}$ of $\mathcal{X}$. Thus $V_{k}$ and $A_{k}$ denote the $k$-skeletons $\mathcal{V}_{k}$ and $\mathcal{X}_{k}$ of $\mathcal{V}$ and $\mathcal{X}$. Similarly, when $\mathcal{V}$ (or $\mathcal{X}$ ) is an abstract stratified set equipped with a system of control data ([8], [18, [19], [35]), we assume given the system of control data of $\mathcal{X}$.

Definition 3.2. Let $\mathcal{X}=(A, \Sigma)$ be a stratified space. A stratified isotopy of $\mathcal{X}$ (or of $A$ ) $\Phi: A \times I \rightarrow A$ (denoted also $\left\{\Phi_{t}: A \rightarrow A\right\}_{t \in I}$ ) is a stratified map such that for every $t \in I$, the map at time $t, \Phi_{t}: A \rightarrow A$, is a stratified homeomorphism of $A$.

Clearly, if $\left\{\Phi_{t}\right\}_{t \in I}$ and $\left\{\Psi_{t}\right\}_{t \in I}$ are stratified isotopies of a stratified space $\mathcal{X}$, then so is $\left\{\Psi_{t} \circ \Phi_{t}\right\}_{t \in I}$.

Definition 3.3. Let $\mathcal{W}=\left(W, \Sigma_{\mathcal{W}}\right)$ and $\mathcal{W}^{\prime}=\left(W^{\prime}, \Sigma_{\mathcal{W}^{\prime}}\right)$ be two substratified objects of a stratified space $\mathcal{X}=(A, \Sigma)$. We say that $W^{\prime}$ is a deformation by isotopy of $W$ in $A$ if there exists a stratified isotopy $\Phi: A \times I \rightarrow A$ such that $\Phi_{0}=1_{A}$ and $W^{\prime}=\Phi_{1}(W)$. We then write $W \stackrel{\Phi}{=} W^{\prime}$.

Clearly "deformation by isotopy" is an equivalence relation on the set of all substratified objects of $\mathcal{X}$.

If $\Phi: A \times I \rightarrow A$ is a stratified isotopy of $\mathcal{X}=(A, \Sigma)$ and $\mathcal{W}$ is a substratified object of $\mathcal{X}$, then for each $t \in I$ the image $\mathcal{W}^{\prime}=\Phi_{t}(\mathcal{W})$ is a substratified object with stratification induced by $\Phi_{t}$, and $W^{\prime}$ is a deformation by isotopy of $W$.

The proof of the main theorem of the section (Theorem 3.8) requires some lemmas.

Lemma 3.4. Let $S$ be a smooth (not necessarily compact) manifold, and $\xi: S \rightarrow$ $T S$ a smooth vector field on $S$.

i) For every proper smooth function $g: S \rightarrow \mathbb{R}$, if $\rho$ denotes the function

$$
\rho: S \rightarrow(0,1], \quad \rho(x)=e^{-\left[g_{* x}(\xi(x))\right]^{2}},
$$

the vector field $\eta(x)=\rho(x) \xi(x)$ is complete (i.e. its flow is defined for every $t \in \mathbb{R}$ ).

ii) If $\xi$ is complete, then for every $C^{\infty}$ function $\delta: S \rightarrow(0,1]$ the vector field $\eta(x)=\delta(x) \xi(x)$ is complete too.

Proof. $i$ ) may be found in [9] (Proposition 1.13, chapter V), while ii) is an exercise.

Lemma 3.5. Given a smooth manifold $S \subseteq \mathbb{R}^{r}$, there exists a family $v_{1}, \ldots, v_{r}$ of $C^{\infty}$ vector fields on $S$ such that:

i) $T_{x} S=\left[v_{1}(x), \ldots, v_{r}(x)\right]$, i.e., $T_{x} S$ is generated by $v_{1}(x), \ldots, v_{r}(x), \forall x \in S$.

ii) $v_{i}$ is complete for each $i \leq r$; i.e., its flow $\phi^{i}: S \times \mathbb{R} \rightarrow S$ is defined $\forall t \in \mathbb{R}$.

iii) If $\partial S=\bar{S}-S \neq \emptyset$, then for every $x_{0} \in \partial S$ one has $\lim _{x \rightarrow x_{0}} v_{i}(x)=0$, with all its derivatives, so that $v_{i} \in C^{\infty}(\bar{S})$ for each $i \leq r$.

Proof. As $S \subseteq \mathbb{R}^{r}$, we can consider the standard constant vector fields $\left\{E_{i}(x)=\right.$ $\left.E_{i}=(0, \ldots, 1, \ldots 0)\right\}_{i \leq r}$ which generate $\mathbb{R}^{r}$ at each point $x$. For each $x \in S$ denote by $p_{T_{x} S}: \mathbb{R}^{r} \rightarrow T_{x} S$ the orthogonal projection of $\mathbb{R}^{r}$ on $T_{x} S$; then, setting $V_{i}(x)=p_{T_{x} S}\left(E_{i}\right)$, where $i \leq r$ and $x \in S$, the vector fields $\left\{V_{i}(x)\right\}_{i \leq r}$ span $T_{x} S$.

Applying Lemma 3.4, we obtain $i)$ and $i i)$ by defining $v_{i}=\rho_{i} V_{i}$ with $\rho_{i}(x)=$ $e^{-\left[g_{* x}\left(V_{i}(x)\right)\right]^{2}}$. 
Now we will show how to choose for each $\rho_{i}(x), i=1, \ldots, r$, the same function $\rho(x)$.

In fact, by reconsidering the proofs of Propositions 1.13 and 1.10 on page 102 of [9], if we set $\rho(x)=e^{-\sum_{j=1}^{r}\left[g_{* x}\left(V_{j}(x)\right)\right]^{2}}$, we see easily that for each maximal integral curve $c:(a, b) \rightarrow S$ of $v_{i}$, for each $i \leq r,\left\|\frac{d}{d t} g \circ c(t)\right\| \leq 1$. Hence $a=-\infty$ and $b=+\infty$; in fact if $b<+\infty$, then $(g \circ c)([0, b))$ would be bounded and $c([0, b)) \subseteq g^{-1}([-M, M])$ would be contained in a compact set of $S$ (as $g$ is proper), implying $b=+\infty$. Similarly, $a=-\infty$. Thus each $v_{i}$ is again complete.

If now $\partial S=\bar{S}-S \neq \emptyset$, then the proof of $i i i)$ follows by considering the vector fields $\left\{v_{i}=\rho V_{i}\right\}_{i<r}$, where $\rho$ is the function

$$
\rho: S \rightarrow(0,1], \quad \rho(x)=e^{-\left(\sum_{j=1}^{r}\left[g_{* x}\left(V_{i}(x)\right)\right]^{2}+\frac{1}{d(x, \partial S)^{2}}\right)}
$$

and $d(x, \partial S)$ denotes the euclidean distance of $x$ from $\partial S$ in $\mathbb{R}^{r}$.

Lemma 3.6. Let $P$ and $Q$ be two smooth submanifolds of a smooth manifold $S$ and let $f$ be a diffeomorphism of $S$. If the graph $\Gamma f$ of $f$ is transverse to $P \times Q$ in $S \times S$, then the restriction $f_{P}: P \rightarrow S$ is transverse to $Q$.

Proof. Exercise.

Definition 3.7. Two substratified objects $\mathcal{V}$ and $\mathcal{W}$ of $\mathcal{X}$ are called transverse over the $k$-skeleton $\mathcal{X}_{k}$ if for each stratum $S$ of $\mathcal{X}_{k}$ the restrictions $\mathcal{V}_{S}$ and $\mathcal{W}_{S}$ are transverse in $S$.

Theorem 3.8 below is an analogue of the transversality theorem stated by Goresky in [12, for a Whitney substratified object $\mathcal{W}$ of $\mathcal{X}$ and a stratified inclusion $i: \mathcal{V} \rightarrow$ $\mathcal{X}$. Here the stratified space $\mathcal{X}$ is an abstract stratified set. We require no further hypothesis about the regularity of the substratified objects of $\mathcal{X}$.

The authors gave a different proof of this theorem in 1997 24] using timedependent vector fields and the techniques introduced by Mather [17] to prove that infinitesimal stability of smooth mappings implies stability.

Theorem 3.8. Let $\mathcal{X}=(A, \Sigma)$ be an abstract stratified set and $\mathcal{V}$ a substratified object of $\mathcal{X}$. For each substratified object $\mathcal{W}$ of $\mathcal{X}$, and each open neighbourhood $U$ of $W$ in $A$, there exists a deformation by isotopy $\mathcal{W}^{\prime}$ of $\mathcal{W}$, transverse to $\mathcal{V}$ in $\mathcal{X}$, such that $W^{\prime} \subseteq U$.

Proof. It will be sufficient to prove the theorem for a $(b)$-regular embedding of $\mathcal{X}=(A, \Sigma)$ in a Euclidean space $\mathbb{R}^{r}$ [1], [27], 31. Suppose then that $A \subseteq \mathbb{R}^{r}$.

By induction on the dimension $k \leq n=\operatorname{dim} A$ of the skeleton $A_{k}$ of $A$, we will construct a chain of $n$ deformations by isotopy of $W$ in $A$,

$$
W(0) \stackrel{\Phi^{1}}{\equiv} W(1) \stackrel{\Phi^{2}}{\equiv} \cdots \stackrel{\Phi^{n}}{\equiv} W(n)
$$

such that $W(k)$ is transverse to $V$ in $A_{k}$ for all $k=0, \ldots, n$.

For $k=0$ we can obviously choose $W(0)=W$.

Suppose we have constructed a chain $W(0) \stackrel{\Phi^{1}}{\equiv} W(1) \stackrel{\Phi^{2}}{\equiv} \ldots \stackrel{\Phi^{k-1}}{\equiv} W(k-1)$.

By the induction hypothesis, $W(k-1)$ is transverse to $V$ in $A_{k-1}$, so we can define the part of $W(k)$ contained in $A_{k-1}$ by

$$
W(k) \cap A_{k-1}=W(k-1) \cap A_{k-1} .
$$


We complete the construction of $W(k)$ in two steps:

Step 1: Construction of $W(k) \cap\left[A_{k}-A_{k-1}\right]$.

Let $S=A_{k}-A_{k-1}$ be the smooth manifold of dimension $k$, the union of all $k$-strata of $A$. We shall find a diffeomorphism $f: S \rightarrow S$ which deforms the strata of $W(k-1) \cap S$, making them transverse to $V \cap S$.

Let us consider a family $v_{1}, \ldots, v_{r}$ of $C^{\infty}$ vector fields on $S$, as constructed in Lemma 3.5, such that:

i) $T_{x} S=\left[v_{1}(x), \ldots, v_{r}(x)\right]$ for every $x \in S$;

ii) $v_{i}$ is complete for each $i \leq r$; i.e. its flow $\phi^{i}: S \times \mathbb{R} \rightarrow S$ is defined $\forall t \in \mathbb{R}$;

iii) $\lim _{x \rightarrow \partial S} v_{i}(x)=0 \quad$ with all its derivatives, so that $v_{i} \in C^{\infty}(\bar{S})$ for each $i \leq r$.

Let us consider the vector field $\zeta$ on $S \times \mathbb{R}^{r}$ defined for $x \in S$ and $b=\left(b_{1}, \ldots, b_{r}\right) \in$ $\mathbb{R}^{r}$ by

$$
\zeta(x, b)=\left(\sum_{i=1}^{r} b_{i} v_{i}(x), 0\right) .
$$

Then $\zeta$ is "level preserving" in the sense that for each $b \in \mathbb{R}^{r}$

$$
\zeta_{b}: S \rightarrow T S, \quad \zeta_{b}(x)=\operatorname{pr}_{1} \zeta(x, b)=\sum_{i=1}^{r} b_{i} v_{i}(x),
$$

defines a vector field tangent to the manifold $S$.

With notation as in Lemma 3.5 , for every $b$ such that $\|b\|<1$ one easily sees that for the maximal integral curves $c_{b}$ of the vector field $\zeta_{b}(x)=\sum_{i=1}^{r} b_{i} \rho(x) V_{i}(x)$, $\left\|\frac{d}{d t} g \circ c_{b}(t)\right\| \leq r$, and this implies that $\zeta_{b}$ has the properties $\left.\left.i\right), i i\right)$ and $\left.i i i\right)$ of Lemma 3.5 .

In particular we deduce that, if $B=B(0,1)$ denotes the open unit ball of $\mathbb{R}^{r}$, for every $b \in B$ the vector field $\zeta_{b}$ has a global flow $\psi^{b}: S \times \mathbb{R} \rightarrow S$ and the same property holds for the restricted vector field (again called $\zeta$ ):

$$
\zeta: S \times B \rightarrow T(S \times B), \quad \zeta(x, b)=\left(\sum_{i=1}^{r} b_{i} v_{i}(x), 0\right) .
$$

If

$$
\psi: S \times B \times \mathbb{R} \rightarrow S \times B, \quad(x, b, t) \mapsto \psi(x, b, t),
$$

denotes the global flow of $\zeta$, then for each $b \in \mathbb{R}^{r}$ the map

$$
\psi^{b}: S \times \mathbb{R} \rightarrow S, \quad \psi^{b}(x, t)=p r_{1} \psi(x, b, t),
$$

is the global flow of the vector field $\zeta_{b}$ on $S$.

Denote by $E_{1}, \ldots, E_{r}$ the standard frame fields of $\mathbb{R}^{r}$ and consider the vector fields $\zeta_{b}$ and $\zeta_{b^{\prime}}$ corresponding to $b=E_{i}$ and $b^{\prime}=\epsilon E_{i}=(0, \ldots, 0, \epsilon, 0, \ldots, 0)$. Such vector fields satisfy:

iv) $\zeta_{E_{i}}(x)=v_{i}(x)$

v) $\zeta_{\epsilon E_{i}}(x)=\epsilon \cdot \zeta_{E_{i}}(x)=\epsilon \cdot v_{i}(x)$, and their flows $\psi^{E_{i}}$ and $\psi^{\epsilon E_{i}}$ are related by the formula

$$
\psi^{\epsilon E_{i}}(x, \tau)=\psi^{E_{i}}(x, \epsilon \tau) .
$$

By considering the smooth map

$$
G: S \times \mathbb{R}^{r} \longrightarrow S, \quad G(x, b)=p r_{1} \circ \psi(x, b, 1)=\psi^{b}(x, 1),
$$

we can prove the submersivity of all partial maps:

$$
G_{x}: \mathbb{R}^{r} \rightarrow S, \quad G_{x}(b)=\psi^{b}(x, 1) .
$$


In fact, for each $x \in S$ we have

$$
\left(G_{x}\right)_{* b}\left(T_{b} \mathbb{R}^{r}\right)=\left(G_{x}\right)_{* b}\left(\left[E_{1}, \ldots, E_{r}\right]\right)=\left[\left(G_{x}\right)_{* b}\left(E_{1}\right), \ldots,\left(G_{x}\right)_{* b}\left(E_{r}\right)\right],
$$

and for each $i \leq r$, we have

$$
\begin{aligned}
\left(G_{x}\right)_{* b}\left(E_{i}\right) & =\left.\frac{\partial G_{x}}{\partial b_{i}}\right|_{b}=\left.\frac{d}{d s}\right|_{s=0} \psi_{1}^{b+s E_{i}}(x)=\left[\left(\psi_{1 * x}^{b+s E_{i}}\right)_{\mid s=0}\left(\left.\frac{d}{d s}\right|_{s=0} \psi^{s E_{i}}(x, 1)\right)\right. \\
& =\psi_{1 * x}^{b}\left(\left.\frac{d}{d s}\right|_{s=0} \psi^{E_{i}}(x, s)\right)=\psi_{1 * x}^{b}\left(\zeta_{E_{i}}(x)\right)=\psi_{1 * x}^{b}\left(v_{i}(x)\right),
\end{aligned}
$$

and, $\psi_{1}^{b}: S \rightarrow S$ being a diffeomorphism, by $\left.i\right)$ we deduce that

$$
\left(G_{x}\right)_{* b}\left(T_{b} \mathbb{R}^{r}\right)=\psi_{1 * x}^{b}\left[v_{1}(x), \ldots, v_{r}(x)\right]=\left[\psi_{1 * x}^{b}\left(v_{1}(x)\right), \ldots, \psi_{1 * x}^{b}\left(v_{r}(x)\right)\right]=T_{x^{\prime}} S,
$$

where $x^{\prime}=G_{x}(b)=\psi^{b}(x, 1)$.

Consider now, for each $b \in B$, the 0 -jet map of $G_{b}=\psi_{1}^{b}: S \rightarrow S$, i.e. the graph map:

$$
j^{0} G_{b}: S \rightarrow S \times S, \quad j^{0} G_{b}(x)=\left(x, G_{b}(x)\right)=\left(x, \psi_{1}^{b}(x)\right),
$$

and denote by $\left\{W_{\alpha}\right\}_{\alpha}$ and by $\left\{V_{\beta}\right\}_{\beta}$ respectively the possibly infinite countable families of strata of the restrictions $W(k-1) \cap S$ and $V \cap S$.

In the same way as in [10] (Lemma 4.6 and Corollary 4.7), by Sard's theorem applied to the map $j: B \rightarrow C^{\infty}(S, S), j(b)=j^{0} G_{b}$, we find that every subset

$$
M_{\alpha, \beta}=\left\{b \in B \quad \mid \quad j^{0} G_{b} \text { is not transverse to } W_{\alpha} \times V_{\beta}\right\} \subseteq B \subseteq \mathbb{R}^{r}
$$

has measure zero in $\mathbb{R}^{r}$, and so this also holds for the countable union $M=$ $\bigcup_{\alpha, \beta} M_{\alpha, \beta}$.

Consider then for a fixed $b \in B-M$ the map $f=\psi_{1}^{b}: S \rightarrow S$.

First of all, by construction we have that $f$ is the time 1 diffeomorphism of the flow of the vector field $\zeta_{b}$. On the other hand, since $b \notin M$, the graph $\Gamma f$ of $f$ is transverse to $W_{\alpha} \times V_{\beta}$, so by Lemma 3.6, $f\left(W_{\alpha}\right)$ is transverse to $W_{\beta}$ for all $\alpha, \beta$, i.e. $f(W(k-1) \cap S)$ is transverse to $V \cap S$.

Hence we can conclude step 1 by defining $W(k) \cap S=f(W(k-1) \cap S)$.

Step 2: Construction of $W(k) \cap\left[A-A_{k}\right]$ and conclusion of the proof.

The aim of this step is to construct a stratified homeomorphism $\tilde{f}: A \rightarrow A$, an extension of $f$, in such a way as to define $W(k) \cap\left[A-A_{k}\right]=\tilde{f}\left(W(k-1) \cap\left[A-A_{k}\right]\right)$. We will not need transversality between $W(k) \cap\left[A-A_{k}\right]$ and $V \cap\left[A-A_{k}\right]$ in $A-A_{k}$.

Since $f=\psi_{1}^{b}$ is the time 1 flow of a vector field $\zeta_{b}$ on $S, \tilde{f}$ will be obtained by extending $\zeta_{b}$ on $A$ and by considering the time 1 flow of such an extension.

We first will do a controlled lifting of $\zeta_{b}$ in a tubular neighbourhood $T_{S}=$ $\bigsqcup_{\operatorname{dim} X=k} T_{X}$ of $S$ and then we modify it slightly to obtain an extension of it on the whole of $A$.

For each $k$-stratum $X$ of $A$, i.e. each connected component $X$ of $S$, choose a tubular neighbourhood $T_{X}=T_{X}(1)$ of $X$ in $A$; these neighbourhoods $\left\{T_{X}\right\}_{X}$ may be chosen pairwise disjoint [19. Denote by $f_{X}: X \rightarrow X$ the restriction of $f$ to $X$.

Clearly $f=\psi_{1}^{b}$ preserves each connected component of $S$, and one can write

$$
f=\bigsqcup_{\operatorname{dim} X=k} f_{X}
$$

with $f_{X} \in \operatorname{Diff}(X, X)$ for each $k$-stratum $X$ of $A$.

Denoting from now on by $\zeta$ the vector field $\zeta_{b}$, similarly we can write $\zeta=\bigsqcup_{X} \zeta_{X}$. 
By Proposition 2.2 there exists a stratified vector field $\zeta_{T_{S}}$, a controlled lifting on $T_{S}=\bigsqcup_{X} T_{X}$ of the vector field $\zeta$ on $S$. As the embedded stratification $\mathcal{X}=(A, \Sigma)$ is $(b)$ - and hence $(c)$-regular, we can also assume that each $\zeta_{T_{S}}$ is continuous on $T_{S}$ ([4], [30], and $\S 2)$.

Because the vector field $\zeta_{T_{S}}$ is a controlled lifting of $\zeta$ and $\zeta$ admits a global flow $\phi=\psi^{b}$, then by Proposition 2.2 again $\zeta_{T_{S}}$ admits a global flow too, namely $\psi_{T_{S}}$, which is a continuous extension of $\phi$.

Let us consider a $C^{\infty}$ function $g: \mathbb{R} \rightarrow[0,1]$ such that $g(s)=1$ if $s \leq \frac{1}{2}$ and $g(s)=0$ if $s \geq 1$. Because the neighbourhoods $\left\{T_{X}\right\}_{\operatorname{dim} X=k}$ are pairwise disjoint, we can define a vector field $\zeta_{A}=\zeta_{A}^{k}$ on the whole of $A$ :

$$
\zeta_{A}(x)=\zeta_{A}^{k}(x)= \begin{cases}g\left(\rho_{X}(x)\right) \cdot \zeta_{T_{X}}(x) & \text { if } x \in \bigsqcup_{\operatorname{dim} X=k} T_{X}(1), \\ 0 & \text { if } x \in A-\bigsqcup_{\operatorname{dim} X=k} T_{X}(1),\end{cases}
$$

which is a continuous extension of $\zeta$, since $\zeta=\zeta_{b}=\sum_{i} b_{i} v_{i}$ satisfies condition iii) of Lemma 3.5.

The vector field $\zeta_{A}$ clearly has a global flow $\Phi: A \times \mathbb{R} \rightarrow A$, which continuously extends the flow of $\zeta_{T_{S}}$ by the identity outside $T_{S}(1)$.

The claimed extension $\tilde{f}: A \rightarrow A$ is found by taking $\tilde{f}=\Phi_{1}: A \rightarrow A$.

In fact, since every $\Phi_{t}: A \rightarrow A$ is a stratified homeomorphism, $\tilde{f}$ is too; since $\zeta_{A}=0$ on $A_{k-1}$, then $\tilde{f}_{\mid A_{k-1}}=1_{A_{k-1}}$; and finally since $f=\psi_{1}^{b}$ (see step 1) and $\zeta_{A}$ extends $\zeta_{b}$, then $\tilde{f}: A \rightarrow A$ extends $f: S \rightarrow S$. Moreover, $\tilde{f}: A \rightarrow A$ is of course a diffeomorphism on each stratum of $A$ and is the identity on $A_{k-1}$. We then define $W(k)=\tilde{f}(W(k-1))$.

Closing induction and end of the proof. As $\Phi$ was constructed in the $k^{\text {th }}$ induction step, we write $\Phi=\Phi^{k}$. Then, as $\Phi_{1 \mid A_{k-1}}^{k}=i d_{A_{k-1}}, \Phi_{1 \mid S}^{k}=f, \Phi_{1}^{k}=\tilde{f}$, one has

$$
W(k)=\left(W(k-1) \cap A_{k-1}\right) \cup f(W(k-1) \cap S) \cup \Phi_{1}^{k}\left(W(k-1) \cap\left(A-A_{k}\right)\right) .
$$

Because $W(k)$ coincides with $W(k-1)$ in $A_{k-1}$ and, by step $1, W(k)$ is transverse to $V$ in $S=A_{k}-A_{k-1}$, we have that $W(k)$ is transverse to $V$ in $A_{k}$.

On the other hand, $W(k)=\Phi_{1}^{k}(W(k-1))$, and so $W(k)$ is a substratified object of $A$ and is a deformation by isotopy of $W(k-1)$.

Finally, "deformation by isotopy" being transitive, we conclude the proof by setting $W^{\prime}=W(n)$. See Remark 3.11 for precise details concerning the neighbourhood $U$ of $W$.

The proof of Theorem 3.8 is based on the idea, sketched on page 39 of the book by Goresky and MacPherson [14, of using a family of self maps of a manifold $S$, following the ingenious method of Abraham and Robbin [1. Step 1 shows in particular the following very useful property: "Every smooth manifold $S$ admits a submersive family of self maps".

A version of this idea can be found in Chapter 6 of the 1976 thesis of Goresky [11.

Theorem 3.9 (Relative version of 3.8). With the same hypotheses and notation as in Theorem 3.8, if $Z$ is a closed subset of $A$ at each point of which $\mathcal{W}$ is transverse to $\mathcal{V}$, one can obtain moreover that the transversalising isotopy $\Phi: A \times I \rightarrow A$ satisfies $\Phi_{t \mid Z}=i d$ for all $t \in I$, and so $\mathcal{W}^{\prime} \cap Z=\mathcal{W} \cap Z$. 
Proof. We adapt the proof of Theorem 3.8 by making a slight modification.

As $Z$ is a closed subset of $A$, there exists a $C^{\infty}$ function $\delta: A \rightarrow[0,1]$ which vanishes only on $Z=\delta^{-1}(0)$. If in the $k^{\text {th }}$ induction step of Theorem 3.8 we replace the family of vector fields $\left\{v_{i}(x),\right\}_{i \leq r}$ by the new family of vector fields $\left\{\omega_{i}(x)=\right.$ $\left.\delta(x) v_{i}(x),\right\}_{i \leq r}$, then properties $\left.\left.\left.i\right), i i\right), i i i\right)$ hold again for $\left\{\omega_{i}\right\}_{i \leq r}$, so the proof of 3.8 gives a complete vector field $\zeta_{A}$ which moreover vanishes on $Z$. Consequently its time 1 flow $\Phi_{1}^{k}: A \rightarrow A$ is a stratified homeomorphism which is the identity map on $Z$.

Thus at each $k^{t h}$ inductive step we obtain

$$
W(k) \cap Z=\Phi_{1}^{k}(W(k-1)) \cap Z=\Phi_{1}^{k}(W(k-1) \cap Z)=W(k-1) \cap Z,
$$

and since this holds for each $k=0, \ldots, n$ (see also Remark 3.10), we can deduce that

$$
W \cap Z=W(0) \cap Z=\cdots=W(n) \cap Z=W^{\prime} \cap Z .
$$

In the remarks below we use the notation of Theorems 3.8 and 3.9.

Remark 3.10. The final deformation $W \stackrel{\Phi}{=} W^{\prime}$, such that $\Phi_{1}(W)$ is transverse to $V$ in $A$, is the composition $\left\{\Phi_{t}=\Phi_{t}^{n} \circ \cdots \circ \Phi_{t}^{1}: A \rightarrow A\right\}_{t \in I}$, where for each $k \leq n, \Phi^{k}$ is a transversalising isotopy in $S=A_{k}-A_{k-1}$ constructed in the $k^{t h}$ step of the induction.

Moreover, $\left\{\Phi_{t}^{k}: A \rightarrow A\right\}_{t \in \mathbb{R}}$ is a one-parameter group of stratified homeomorphisms of $A$, the flow of the stratified vector field $\zeta_{A}^{k}(x)$ on $A$.

Remark 3.11. The map $f=\psi_{1}^{b}: S \rightarrow S$, transversalising in $S=A_{k}-A_{k-1}$, is obtained as the time 1 flow of the vector field $\zeta_{b}(x)=\sum_{i=1}^{r} b_{i} v_{i}(x)$ on $S$ whose parameter $b=\left(b_{1}, \ldots, b_{r}\right) \in B=B(0,1)$ is chosen outside a measure zero set $M \subseteq B(0,1) \subseteq \mathbb{R}^{r}$. Since $b$ may be chosen arbitrarily close to 0 in $\mathbb{R}^{r}$, the vector field $\zeta_{b}$ on $S$ may be taken to be arbitrarily small and its global flow $\left\{\psi_{t}^{b}: S \rightarrow S\right\}_{t \in \mathbb{R}}$ becomes arbitrarily close to the identity map $1_{S}$. The same arguments apply for each $k \leq n$ to the extension $\Phi_{t}^{k}: A \rightarrow A$ of the $k^{t h}$ inductive step, and hence for the final transversalising extension $\Phi_{t}=\Phi_{t}^{n} \circ \cdots \circ \Phi_{t}^{1}: A \rightarrow A$.

In conclusion, as stated in Theorem 3.8, the transversalising stratified homeomorphism $\Phi_{1}: A \rightarrow A$ may be constructed so that $W^{\prime}=\Phi_{1}(W)$ lies in an arbitrarily small neighbourhood $U$ of $W$ in $A$.

The $(b)-,(c)-,(w)$ - and $(L)$-regular cases.

Remark 3.12. K. Bekka has shown [2, 3] that (c)-regular stratifications admit a system of control data; so both $(b)$-regular and $(c)$-regular stratified sets are abstract stratified sets, and hence Theorems 3.8 and 3.9 hold for them.

If $\mathcal{X}$ is a $(w)$-regular or $(L)$-regular stratification, it has particularly good lifting of vector fields 6], 29] assuring important properties such as local topological triviality [29], 34], but may have no system of control data defining an abstract stratified structure. Therefore the statement in Theorem 3.8 is not convenient for such stratifications. Fortunately the proofs of Theorems 3.8 and 3.9 are more general than their statements, and still work with a slight modification.

If $\mathcal{X}$ is a subanalytic $(w)$-regular stratification of a subanalytic set, then it does admit a system of control data, because it is then (b)-regular [15], [34]. This is also the case for definable sets in o-minimal structures [16]. 
Theorem 3.13. Let $\mathcal{X}=(A, \Sigma)$ be a $(w)$-regular stratification of a closed subset of a smooth manifold $M$, and let $\mathcal{V}$ be a substratified object of $\mathcal{X}$. Given a substratified object $\mathcal{W}$ of $\mathcal{X}$, and an open neighbourhood $U$ of $\mathcal{W}$ in $A$, there exists a deformation by isotopy $\mathcal{W}^{\prime}$ of $\mathcal{W}$ which is transverse to $\mathcal{V}$ in $\mathcal{X}$ and such that $W^{\prime} \subseteq U$.

Moreover if $Z$ is a closed subset of $A$ at each point of which $\mathcal{W}$ is transverse to $\mathcal{V}$, one can obtain $\mathcal{W}^{\prime} \cap Z=\mathcal{W} \cap Z$.

Proof. The proof is almost the same as that of Theorem 3.8.

The same proof works for step 1 . In step 2 , to define "the continuation" $W(k) \cap$ $\left(A-A_{k}\right)$, we extend the transversalising diffeomorphism $f: S \rightarrow S$ to higher strata so as to obtain a stratified homeomorphism of $A$ which is a diffeomorphism of each stratum. This is possible because every vector field having a global flow on a $(w)$-regular stratification admits a stratified $(w)$-regular lifting on the higher strata having a global flow (not necessarily controlled in this case) [34].

Remark 3.14. Because $(L)$-regular stratifications are $(w)$-regular [29], Theorem 3.15 holds also for Mostowski's $(L)$-regular stratified spaces.

Remark 3.15 . Remarks 3.10 and 3.11 apply also to $(w)$-regular stratifications.

One can ask: "Is there a deformation $W^{\prime}$ of $W$ such that $W^{\prime} \cap V$ and $W^{\prime} \cup V$ have induced natural stratifications?" That is, denoting by $C(H)$ the connected components of a space $H$, take the partition in smooth manifolds of $W^{\prime} \cup V$ defined by

$$
\Sigma_{W^{\prime} \cup V}=\bigsqcup_{W_{\alpha}^{\prime} \subseteq W^{\prime}, V_{\beta} \subseteq W}\left(C\left(W_{\alpha}^{\prime}-V_{\beta}\right) \sqcup C\left(V_{\beta}-W_{\alpha}^{\prime}\right) \sqcup C\left(W_{\alpha}^{\prime} \cap V_{\beta}\right)\right)
$$

and consider the following questions:

i) Is there a deformation by isotopy $W^{\prime}$ of $W$ such that the partition $\Sigma_{W^{\prime} \cup V}$ of manifolds satisfies the frontier condition?

ii) Is there a deformation $W^{\prime}$ of $W$ transverse to $V$ such that for each pair of strata $W_{\alpha}^{\prime}, V_{\beta}$ of $W^{\prime}$ and $V$ respectively, the intersection $W_{\alpha}^{\prime} \cap V_{\beta}$ is locally finite in $W^{\prime} \cup V$ ?

If so, then $\Sigma_{W^{\prime} \cup V}$ would be the natural induced stratification of $W^{\prime} \cup V$, and

$$
\Sigma_{W^{\prime} \cap V}=\bigsqcup_{\alpha, \beta} C\left(W_{\alpha}^{\prime} \cap V_{\beta}\right)
$$

would be the natural stratification of $W^{\prime} \cap V$.

Figures 1 and 2 show simple examples of substratified objects $\mathcal{V}$ and $\mathcal{W}$ transverse in $\mathcal{X}$ for which the difficulties i) and ii) are present. In Figure 1 the "frontier condition" fails, while in Figure 2 "local finiteness" fails.

We conclude the section by showing, in Examples 3.16 and 3.17, that the answer to both questions $i$ ) and ii) is no.

Example 3.16. The answer to question $i$ ) is no, i.e. it is not possible to obtain in general that $\mathcal{W} \cap_{t} \mathcal{V}$ or $\mathcal{W} \cup_{t} \mathcal{V}$ satisfies the frontier condition.

Proof. Let us consider as ambient stratification $\mathcal{X}$ the Euclidean space $\mathbb{R}^{3}$ stratified with two strata by $\Sigma=\left\{X=S^{1} \times\{0\}, Y=\mathbb{R}^{3}-X\right\}$, where $S^{1}=\{(x, y) \in$ $\left.\mathbb{R}^{2} \mid x^{2}+y^{2}=1\right\}$ is the unit circle of the $(x, y)$-plane. Choose as substratified object 


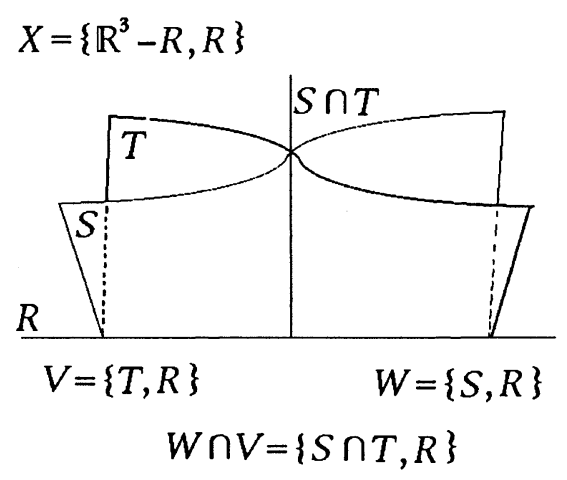

FiguRE 1.

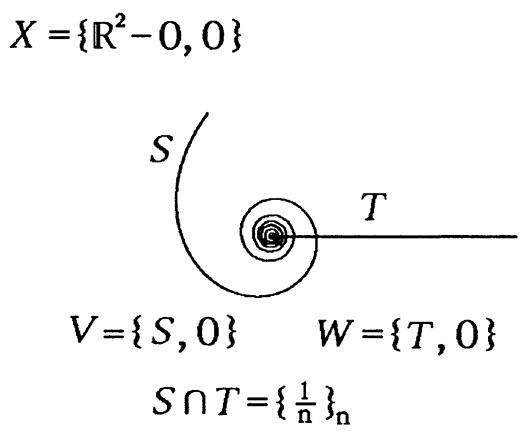

FiguRE 2.

$\mathcal{W}$ of $\mathcal{X}$ the cylinder $W=S^{1} \times[0, \infty)$ stratified with two strata by $\Sigma_{\mathcal{W}}=\{X=$ $\left.S^{1} \times\{0\}, C=S^{1} \times(0, \infty)\right\}$, and take for $\mathcal{V}$ the closed Moebius band $M$ obtained by a half rotation around the circle $X$ of the closed segment $\overline{Q P}$ with $Q=\left(0,-1,-\frac{1}{4}\right)$, and $P=\left(0,-1, \frac{1}{4}\right)$, and stratified by $\Sigma_{\mathcal{V}}=\{X, \partial M, M-(X \cup \partial M)\}$.

Then $\mathcal{W} \cap X=X$ and $\mathcal{V} \cap X=X$ are obviously transverse in $X$, and after a slight deformation near $P, \mathcal{W} \cap Y$ and $\mathcal{V} \cap Y$ are transverse in $Y$, so $\mathcal{W}$ and $\mathcal{V}$ are transverse in $\mathcal{X}$. On the other hand, as $C \cap \partial M=\{P\}$ and $C \cap(M-(X \cup \partial M))=$ the open segment $Q^{\prime} P$ with $Q^{\prime}=(0,-1,0)$, then $\mathcal{W} \cap_{t} \mathcal{V}=\left\{X, Q^{\prime} P,\{P\}=C \cap \partial M\right\}$ does not satisfy the frontier condition, because the two strata $X$ and $Q^{\prime} P$ do not. Moreover, for any other (transversalising) deformation by isotopy $\mathcal{W}^{\prime}$ of $\mathcal{W}$ the same obstruction appears.

Example 3.17. The answer to question ii) is no. Thus our result is the best possible.

Proof. Let us consider for $\mathcal{X}$ the Euclidean space $\mathbb{R}^{3}$ stratified by the two strata $\Sigma=\left\{X=x\right.$-axis, $\left.Y=\mathbb{R}^{3}-X\right\}$.

To define two convenient substratified objects $\mathcal{W}$ and $\mathcal{V}$ of $\mathcal{X}$, consider an open spiral $S$ of the coordinate plane $(y, z)$, turning infinitely many times around the origin, defined in the polar coordinates of the plane $(y, z)$ by $S=\{(\rho, \theta) \mid \rho=$ $\left.h(\theta), \theta \in\left(\frac{\pi}{2}, \infty\right)\right\}$, where $h(\theta)$ is a positive strictly decreasing function satisfying 


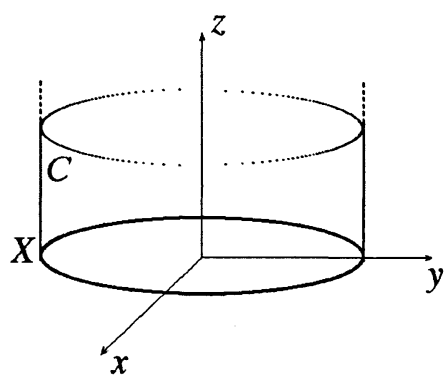

$W=X \cup C$

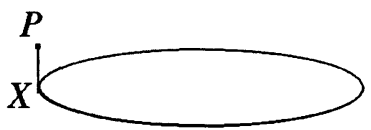

$w \cap V$

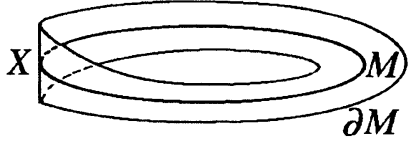

$V=M-X-\partial M$

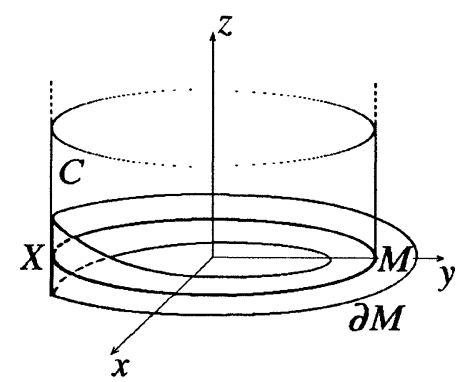

$W \cup V$

FiguRE 3.

$\lim _{\theta \rightarrow \infty} h(\theta)=0$ (choosing $h(\theta)$ appropriately, one can obtain spirals with a desired type of regularity).

Let us consider for $\mathcal{W}$ the regular substratified object of $\mathcal{X}$ whose support is the product $\mathbb{R} \times \bar{S} \subseteq \mathbb{R}^{3}$ stratified by $\Sigma_{\mathcal{W}}=\left\{X=\mathbb{R} \times 0^{2}, \alpha=\mathbb{R} \times S\right\}$ (which inherits the same regularity as $S$ ).

To define a convenient substratified object $\mathcal{V}$ of $\mathcal{X}$, we first define a "double spiralling" curve $S^{\prime}$. Take a closed tubular neighbourhood $N(S)$ of $S$ of strictly decreasing radius $r$ tending to 0 near the origin, and starting from a point in $\partial N(S)$ consider a spiral $S^{\prime}$ in $\partial N(S)$ turning infinitely many times around $S$ (one can also obtain without much difficulty a given regularity for $S^{\prime}$ ).

The spiral $S^{\prime}$ has the following special property: for every plane $\alpha^{\prime}$ containing the origin $O, \alpha^{\prime} \cap S^{\prime}$ contains an infinite sequence of points which accumulate near $O$, and the same property holds by taking for $\alpha^{\prime}$ (a germ of) an open half-plane near 0 .

In fact, since $\alpha^{\prime} \cap S$ is an infinite set of points $\left\{d_{n}\right\}_{n}$ accumulating near $O$, then $\alpha^{\prime} \cap N(S)=\bigsqcup_{n} D_{n}$ is an infinite union of pairwise disjoint topological closed balls $D_{n}$, with $d_{n} \in D_{n}^{o}$, and on the boundary of each $D_{n}$ lies a point $c_{n}$ of the double spiral $S^{\prime}$. Thus $S^{\prime} \cap \alpha^{\prime} \supseteq \bigcup_{n}\left\{c_{n}\right\}$, and the sequence $\left\{c_{n}\right\}_{n}$ accumulates near $O$.

Take for $\mathcal{V}$ the substratified object of $\mathcal{X}$ stratified by $\Sigma_{\mathcal{V}}=\left\{O, S^{\prime}, S^{\prime \prime}\right\}$, where $S^{\prime \prime}=\{t u \mid t \in(0, \infty)\}$, with $u \in \mathbb{R}^{3}-S$ an arbitrary open half-line of $\mathbb{R}^{3}-S$ 

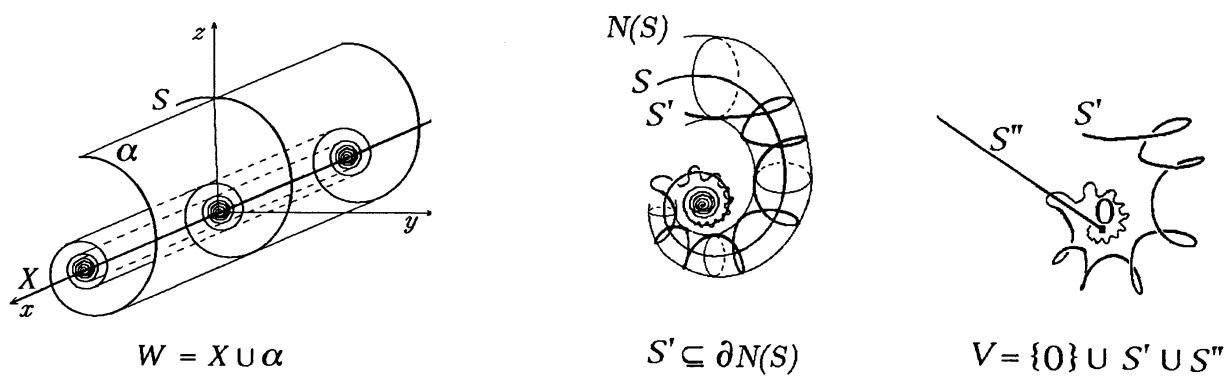

FIgURE 4.

not meeting $S^{\prime}$ (for this it suffices to choose $S^{\prime \prime}$ not meeting $N(S)$, because $S^{\prime} \subseteq$ $\partial N(S) \subseteq N(S))$.

In this setting there is no deformation by isotopy $\mathcal{W}^{\prime}=\Phi_{1}(\mathcal{W})$ of $\mathcal{W}$ for which $\mathcal{W}^{\prime} \cap_{t} \mathcal{V}$ is locally finite. In fact, in the lower stratum $X$ of $\mathcal{X}$ we have $\mathcal{W} \cap X=X$ and $\mathcal{V} \cap X=\{0\}$, so $\mathcal{W}$ and $\mathcal{V}$ are transverse in $X$. In the higher stratum $Y=\mathbb{R}^{3}-X$ of $\mathcal{X}$ we have

$$
\mathcal{W} \cap \mathcal{V} \supseteq \alpha \cap S^{\prime \prime}
$$

and hence $\mathcal{W} \cap \mathcal{V}$ is clearly not locally finite, and moreover every deformation $\Phi_{1}$ of $Y=\mathbb{R}^{3}-X$, putting $\alpha$ in a position of locally finite intersection with $S^{\prime \prime}$ near $O$, has to deform $\alpha$ into a germ of a half-plane $\alpha^{\prime}$, necessarily meeting the double spiral $S^{\prime}$ in an intersection which is not locally finite. Then for every deformation by isotopy $\mathcal{W}^{\prime}$ of $\mathcal{W}, \mathcal{W}^{\prime} \cap \mathcal{V}$ is not locally finite, and this also automatically holds for $\mathcal{W}^{\prime} \cup \mathcal{V}$.

Examples 3.16 and 3.17, show that transversality Theorems 3.8, 3.9 and 3.13 are the best possible results in the stratified $C^{\infty}$ category.

\section{Transversality OF STRATIFIED MAPS}

In this section we generalise our previous results to transversality of stratified maps.

In this stratified transversality theorem (Theorem 4.4) we deform a stratified map $h: \mathcal{Z} \rightarrow \mathcal{X}$ to make it transverse to a fixed stratified map $g: \mathcal{Y} \rightarrow \mathcal{X}$.

Definition 4.1. For a pair of stratified spaces $\mathcal{Y}, \mathcal{X}$ denote by $\operatorname{SM}(\mathcal{Y}, \mathcal{X})$ the set of stratified maps $h: \mathcal{Y} \rightarrow \mathcal{X}$. Let $h, h^{\prime}: \mathcal{Y} \rightarrow \mathcal{X}$ be two elements of $\operatorname{SM}(\mathcal{Y}, \mathcal{X})$. We say that $h^{\prime}$ is a deformation by isotopy of $h$ in $\mathcal{X}$ when there exists a stratified isotopy $\Phi: \mathcal{X} \times I \rightarrow \mathcal{X}$ such that $\Phi_{0}=1_{\mathcal{X}}$ and $h^{\prime}=\Phi_{1} \circ h$, i.e. $h^{\prime}$ is the deformation via $\Phi$ and at time $t=1$ of $h$. Then we write $h \stackrel{\Phi}{=} h^{\prime}$.

Deformation by isotopy in $\mathcal{X}$ is an equivalence relation on the set $\operatorname{SM}(\mathcal{Y}, \mathcal{X})$.

If $\Phi: A \times I \rightarrow A$ is a stratified isotopy of $\mathcal{X}=(A, \Sigma)$ and $h \in \operatorname{SM}(\mathcal{Y}, \mathcal{X})$, then for each $t \in I$ the map $h_{t}=\Phi_{t} \circ h \in \operatorname{SM}(\mathcal{Y}, \mathcal{X})$ is a deformation by isotopy of $h$ in $\mathcal{X}$.

Definition 4.2. Two maps $h, g \in \operatorname{SM}(\mathcal{Y}, \mathcal{X})$ are said to be transverse over the $k$-skeleton $\mathcal{X}_{k}$ (or $A_{k}$ ) of $\mathcal{X}$ when for each stratum $S$ of $\mathcal{X}_{k}$ their restrictions to $S$ are transverse in $S$. More precisely, given strata $P$ and $Q$ of $\mathcal{Y}$ such that $h(P) \subseteq S$ and $g(Q) \subseteq S$, the restrictions $h_{\mid P}: P \rightarrow S$ and $g_{\mid Q}: Q \rightarrow S$ are transverse. 
The lemma below is the version for maps of Lemma 3.5, and is easy to prove:

Lemma 4.3. Let $S$ be a $C^{1}$ manifold, let $f \in C^{1}(S, S)$, and let $h: P \rightarrow S$ and $g: Q \rightarrow S$ be $C^{1}$ maps defined on $C^{1}$ manifolds $P$ and $Q$.

If the graph $\Gamma f$ of $f$ is transverse to $(h \times g)$, then $f \circ h$ is transverse to $g$.

We can now state

Theorem 4.4. Let $\mathcal{X}=(A, \Sigma)$ be an abstract stratified set, or a $(w)$-regular stratified subset of a manifold, and let $g: \mathcal{Y} \rightarrow \mathcal{X}$ be a stratified map.

Then for each stratified map $h: \mathcal{Z} \rightarrow \mathcal{X}$ and each open neighbourhood $U$ of $h(\mathcal{Z})$ in $\mathcal{X}$, there exists a deformation by isotopy $h^{\prime}$ of $h$ in $\mathcal{X}$ which is transverse to $g$ in $\mathcal{X}$ and such that $h^{\prime}(\mathcal{Z}) \subseteq U$. If $C$ is a closed subset of $\mathcal{X}$ on which $h$ is transverse to $g$, then one can obtain that $h^{\prime}=h$ on $C$.

Proof. We adapt the proof of Theorem 3.8. By induction on the dimension $k \leq$ $n=\operatorname{dim} \mathcal{X}$ of the $k$-skeleton $A_{k}$ of $A$ we construct a chain of $n$ deformations by isotopy of $h$ :

$$
h=h^{0} \stackrel{\Phi^{1}}{=} h^{1} \stackrel{\Phi^{2}}{\equiv} \cdots \stackrel{\Phi^{n}}{\equiv} h^{n}=h^{\prime}
$$

such that $h^{k}$ is transverse to $g$ in $A_{k}$ for every $k=0, \ldots, n$, and $h_{\mid C}^{k}=h_{\mid C}$.

Let $k>0$ and suppose that a chain $h^{0} \stackrel{\Phi^{1}}{\equiv} h^{1} \stackrel{\Phi^{2}}{\equiv} \ldots \stackrel{\Phi^{k-1}}{\equiv} h^{k-1}$ has been constructed.

By the inductive hypothesis $h^{k-1}$ is transverse to $g$ in $A_{k-1}$, so we define the restriction of $h^{k}$ to $A_{k-1}$ to coincide with $h^{k-1}$. As in Theorem 3.8, we complete the proof by deforming $h^{k-1}$ without changing it on $A_{k-1}$ and $C$.

Step 1 : Deformation of $h_{\mid\left[A_{k}-A_{k-1}\right]}^{k-1}$.

We can suppose that $S=A_{k}-A_{k-1}$ is the unique connected $k$-stratum of $\mathcal{X}$.

Consider the family $\left\{W_{\alpha}\right\}_{\alpha}$ of all strata of $\left(h^{k-1}\right)^{-1}(S)$, and for each $\alpha$ let $h_{\alpha}: W_{\alpha} \rightarrow S$ be the restriction of $h$ to $W_{\alpha}$. Similarly, write $\left\{Y_{\beta}\right\}_{\beta}$ for the strata of $g^{-1}(S)$ and $g_{\beta}: Y_{\beta} \rightarrow S$ for the restriction of $g$ to $Y_{\beta}$ for each $\beta$.

Step 1 may be deduced in a way analogous to step 1 of Theorems 3.8 and 3.9, where we replace the restrictions $\mathcal{W}_{S}$ and $\mathcal{V}_{S}$ of the substratified objects $\mathcal{W}$ and $\mathcal{V}$ by the restrictions $h_{S}$ and $g_{S}$ of the maps $h$ and $g$. If we denote by $j^{0} f$ the graph map of $f$, then the set

$$
M_{\alpha, \beta}=\left\{b \in B \mid j^{0} f \text { is not transverse to }\left(h_{\alpha} \times g_{\beta}\right)\right\}
$$

has measure zero in $\mathbb{R}^{n}$, so the set $M=\bigcup_{\alpha, \beta} M_{\alpha, \beta}$ has measure zero too.

With the same notation as in 3.8 , choose $b \in B-M$ and consider the diffeomorphism $f=\Psi_{1}^{b}: S \rightarrow S$.

Lemma 4.3 implies that $f \circ h_{\alpha}^{k-1}$ is transverse to $g_{\beta}$ for every $\alpha, \beta$, and so $f \circ h_{S}^{k-1}$ is transverse to $g_{S}$. We then define $h^{k}=f \circ h^{k-1}$, so $h_{S}^{k}$ is transverse to $g_{S}$.

Also $f=i d$ on $A_{k-1}$, so $h^{k}$ and $h^{k-1}$ coincide on $A_{k-1}$, and (by the inductive hypothesis on $\left.h^{k-1}\right) f \circ h_{A_{k-1}}^{k}$ is transverse to $g_{A_{k-1}}$ in $A_{k-1}$. Therefore $f \circ h_{A_{k}}^{k}$ is transverse to $g_{A_{k}}$ on $A_{k}=A_{k-1} \cup S$, and this concludes the proof of step 1 . 
Step 2: Deformation of $h_{\left[A-A_{k}\right]}^{k-1}$ and conclusion of the proof.

In a similar way to step 2 of the proof of Theorem 3.8, using the same stratified homeomorphism $\tilde{f}=\Phi_{1}^{k}: A \rightarrow A$ we set $h^{k}=\tilde{f} \circ h^{k-1}$.

The stratified isotopy $\Phi^{k}$ satisfying $\Phi_{1 \mid A_{k-1}}^{k}=i d_{A_{k-1}}, \quad \Phi_{1 \mid S}^{k}=f, \quad \Phi_{1}^{k}=\tilde{f}$, we deduce the "whole deformation" $h^{k}$ of $h^{k-1}$ by defining $h^{k}=\Phi_{1}^{k} \circ h^{k-1}$.

In fact we have

$$
h^{k}= \begin{cases}h_{A_{k-1}-1}^{k-1} & \text { on } A_{k-1}, \\ f \circ h_{S}^{k-1} & \text { on } S=A_{k}-A_{k-1}, \\ \Phi_{1}^{k} \circ h_{\left[A-A_{k}\right]}^{k-1} & \text { on }\left[A-A_{k}\right],\end{cases}
$$

and this concludes the inductive step.

We complete the proof of the theorem by setting $h^{\prime}=h^{n}$. Then $h_{\mid C}^{\prime}=h_{\mid C}$.

Suppose now, as in the transversality lemma of Goresky, that $\mathcal{W}$ is a substratified object of $\mathcal{X}$, and that the map $h=i: \mathcal{W} \hookrightarrow \mathcal{X}$ is the stratified inclusion of $\mathcal{W}$ in $\mathcal{X}$, and consider the map $h^{\prime}=\Phi_{1} \circ h$. Because the transversalising deformation $\Phi_{1}$ is a stratified homeomorphism, and hence is a diffeomorphism on each stratum, by Lemma 4.3 the condition " $h$ ' $=\Phi_{1} \circ h$ is transverse to $g$ " may be reread as ' $\mathcal{W} '=\Phi_{1}(\mathcal{W})$ is transverse to $g$ '. Thus we have the following corollary, which generalizes the transversality lemma of Goresky, without the $\pi$-fibre condition on the substratified object $\mathcal{W}$ to be deformed.

Corollary 4.5. Let $\mathcal{X}$ be an abstract stratified set, or a $(w)$-regular stratified subspace of a manifold, and let $g: \mathcal{Y} \rightarrow \mathcal{X}$ be a stratified map on a stratified space $\mathcal{Y}$.

Then for each substratified object $\mathcal{W}$ of $\mathcal{X}$ and each open neighbourhood $U$ of $W$ in $\mathcal{X}$, there exists a deformation by isotopy $\mathcal{W}^{\prime}$ of $\mathcal{W}$ which is transverse to $g$ and such that $W^{\prime} \subseteq U$. Moreover, if $C$ is a closed subset of $\mathcal{X}$ on which $\mathcal{W}$ is transverse to $g$, then we can obtain that $\mathcal{W}^{\prime} \cap C=\mathcal{W} \cap C$.

Proof. An immediate application of Theorem 4.4.

Remark 4.6. The analogies of Remarks 3.10, 3.11, 3.12, 3.14, and 3.15 concerning respectively Theorems 3.8, 3.9 and 3.13 apply also to Theorem 4.4 and Corollary 4.5 .

Corollary 4.5 holds for stratifications and stratified maps that are more general than those of the transversality lemma of [12. For we do not require

i) that $g$ be controlled with respect to two fixed systems of control data $\mathcal{T}_{1}$ and $\mathcal{T}_{2}$ respectively of $\mathcal{Y}$ and $\mathcal{X}$, or that $g$ be the restriction of a smooth map $\tilde{g}: M_{1} \rightarrow M_{2}$ between two smooth manifolds containing respectively $\mathcal{Y}$ and $\mathcal{X}$;

or

ii) that $\mathcal{W}$ satisfy the $\pi$-fibre condition.

The $\pi$-fibre condition is a very strong restriction on the geometry of the substratified object $\mathcal{W}$ of $\mathcal{X}$, and ensures that $(b)$-regularity is preserved, as was shown in 12; possibly other regularity conditions are preserved. For example, this is the case for $(a)$-regularity, but it could also be true for $(w)$-regularity or $(c)$-regularity.

In Corollary 4.5, as we do not consider any regularity condition for $\mathcal{W}$ other than being a substratified object of $\mathcal{X}$, the problem of the preservation of such a condition by deformation by isotopy does not arise. We devote the next section to this delicate problem. 


\section{Preservation of Regularity \\ FOR THE DEFORMED SUBSTRATIFIED OBJECTS}

If the transversalising stratified isotopy of Theorem 3.8 is "semi-differentiable" (a notion intermediate between continuity and differentiability for stratified maps due to the first author [24]), the transversalising stratified homeomorphism $\Phi_{1}: \mathcal{X} \rightarrow \mathcal{X}$ preserves $(a)$ - and $(c)$-regularity of the substratified objects of $\mathcal{X}$ as follows.

Recall that conditions $(a)$ and $(b)$ of Whitney, and $(c)$ of Bekka, are preserved by $C^{1}$-diffeomorphisms [33], [3].

Definition 5.1. Let $\mathcal{X}$ and $\mathcal{X}^{\prime}$ be $(a)$-regular stratified spaces in smooth manifolds $N$ and $M$ respectively. A stratified map $f: \mathcal{X} \rightarrow \mathcal{X}^{\prime}$ is called semi-differentiable at $x \in X$ iff for every stratum $Y>X(\bar{Y} \supseteq X)$ and for each sequence $\left\{\left(y_{n}, v_{n}\right)\right\}_{n} \subseteq$ $T Y$ converging to a point $(x, v) \in T X$ we also have $\lim _{n \rightarrow \infty} f_{Y * y_{n}}\left(v_{n}\right)=f_{X * x}(v)$.

Since $f$ is a stratified map, there exist strata $X^{\prime}$ and $Y^{\prime}$ of $\mathcal{X}^{\prime}$ such that $f(X) \subseteq$ $X^{\prime}$ and $f(Y) \subseteq Y^{\prime}$, and moreover $Y^{\prime} \geq X^{\prime}$ by continuity of $f$. Then by the frontier condition $X \subseteq \bar{Y} \subseteq N$ and $(a)$-regularity, of $\mathcal{Y}$ and $\mathcal{X}$ it follows that $T X \subseteq \overline{T Y}$ and $T X^{\prime} \subseteq \overline{T Y^{\prime}}$ with the topology of $T N$ and $T M$ respectively.

A map $f$ is semi-differentiable on a stratum $X$ (resp. on the whole of $\mathcal{X}$ ) if it is semi-differentiable at each $x \in X$ (resp. for every stratum $X$ of $\mathcal{X}$ ) [24], 25].

If the stratified maps $g: \mathcal{Z} \rightarrow \mathcal{Y}$ and $f: \mathcal{Y} \rightarrow \mathcal{X}$ are semi-differentiable, then $f \circ g: \mathcal{Z} \rightarrow \mathcal{X}$ is semi-differentiable. A detailed account of semi-differentiability and other similar conditions on stratified maps may be found in [24].

Proposition 5.2. Let $\mathcal{X}=(A, \Sigma)$ be a $(c)$-regular stratified space, and for the isotopy $\Phi: A \times I \rightarrow A$ satisfying $\mathcal{W}^{\prime}=\Phi_{1}(\mathcal{W})$ let $\Phi_{1}$ be semi-differentiable. Then

i) $\mathcal{W}^{\prime}$ is $(c)$-regular if $\mathcal{W}$ is $(c)$-regular, and

ii) $\mathcal{W}^{\prime}$ is (a)-regular if $\mathcal{W}$ is (a)-regular.

Proof. For every stratum $R$ of $\mathcal{W}$ whose distance function is $\rho_{R}: T_{R} \rightarrow[0, \infty[$, let $R^{\prime}=\Phi_{1}(R)$ be the corresponding stratum of $\mathcal{W}^{\prime}$ and consider for $R^{\prime}$ the distance function $\rho_{R^{\prime}}=\rho_{R} \circ \Phi_{1}^{-1}: T_{R^{\prime}}=\Phi_{1}\left(T_{R}\right) \rightarrow[0, \infty[$. Then the proof of $i$ ) reduces to a simple verification, and a similar argument works for $i i)$.

Note that the distance function $\rho_{R^{\prime}}=\rho_{R} \circ \Phi_{1}^{-1}$ need not be $C^{1}$, but it is continuous. Therefore in statement $i$ ) of Proposition 5.2, (c)-regularity is weaker than that of Bekka: take distance functions $\left\{\rho_{R}: T_{R} \rightarrow[0, \infty)\right\}_{R}$, smooth on each intersection $T_{R S}=T_{R} \cap S$ with $S \geq R$, and merely continuous on $T_{R}$. However, one can see that such a slight change (with respect to the original definition of (c)-regularity) does not modify the validity of the property of continuous lifting of vector fields, nor that of the first isotopy theorem.

As mentioned in the introduction, when $\mathcal{X}$ has a Whitney (b)-regular stratification, to our knowledge it remains unknown whether semi-differentiability of $\Phi_{1}: \mathcal{X} \rightarrow \mathcal{X}$ suffices to ensure preservation of $(b)$-regularity.

\section{REFERENCES}

1. R. Abraham and J. Robbin, Transversal mappings and flows, W.A. Benjamin, Inc, New York, 1967. MR 39:2181

2. K. Bekka, Sur les propriétés topologiques et métriques des espaces stratifiés, thesis, University of Paris-Sud, Orsay, 1988.

3. K. Bekka, C-régularité et trivialité topologique, Singularity theory and its applications, Warwick 1989, Part I, Lecture Notes in Math. 1462, Springer, Berlin, 1991, 42-62. MR 92h:58012 
4. K. Bekka, Isotopy theorem, preprint, University of Liverpool, 1991.

5. K. Bekka and C. Murolo, Homologie d'espaces stratifiés, C. R. Acad. Sci. Paris, t. 331, Série I, p. 703-708, 2000. MR 2001m:57064

6. H. Brodersen and D. J. A. Trotman, Whitney (b)-regularity is weaker than Kuo's ratio test for real algebraic stratifications, Math. Scand. 45 (1979), 27-34. MR 81i:58008

7. W. Fulton, Intersection Theory, Springer Verlag, Berlin-Heidelberg, 1984. MR 85k:14004

8. C. G. Gibson, K. Wirthmüller, A. A. du Plessis and E. J. N. Looijenga, Topological stability of smooth mappings, Lecture Notes in Math. 552, Springer-Verlag, 1976. MR 55:9151

9. C. Godbillon, Géométrie Différentielle et Mécanique Analytique, Hermann, Paris 1969. MR 39:3416

10. M. Golubitsky and V. Guillemin, Stable mappings and their singularities, Graduate Texts in Mathematics 14, Springer-Verlag, Berlin and New York, 1973. MR 49:6269

11. M. Goresky, Cohomology and homology of stratified objects, thesis, Brown University, 1976.

12. M. Goresky, Whitney stratified chains and cochains, Trans. Amer. Math. Soc. 267 (1981), 175-196. MR 82j:58008

13. M. Goresky and R. MacPherson, Intersection homology theory, Topology 19 (1980), 135-162. MR 82b:57010

14. M. Goresky and R. MacPherson, Stratified Morse theory, Springer-Verlag, Berlin, 1987. MR 90d:57039

15. T.-C. Kuo, The ratio test for analytic Whitney stratifications, Proc. of Liverpool Singularities Symposium I, Lecture Notes in Math. 192, Springer (1971), 141-149. MR 43:5056

16. Ta Lê Loi, Verdier and strict Thom stratifications in o-minimal structures, Illinois Journal of Math. 42 (1998), 347-356. MR 99c:32058

17. J. Mather, Stability of $C^{\infty}$ mappings: II. Infinitesimal stability implies stability, Annals of Mathematics 89 (1969), 254-291. MR 41:4582

18. J. Mather, Stratifications and mappings, Dynamical Systems (M. Peixoto, Editor), Academic Press, New York, 1971, 195-223. MR 51:4306

19. J. Mather, Notes on topological stability, Mimeographed notes, Harvard University, 1970.

20. C. McCrory, Poincaré duality in spaces with singularities, Thesis, Brandeis University, 1972.

21. C. McCrory, Stratified general position, Algebraic and Geometric Topology, Santa Barbara 1977, Lecture Notes in Math. 664, Springer-Verlag, Berlin and New York, 1978, 142-146. MR 80m:57016

22. C. Murolo, Whitney homology, cohomology and Steenrod squares, Ricerche di Matematica 43 (1994), 175-204. MR 96a:55012

23. C. Murolo, The Steenrod p-powers in Whitney cohomology, Topology and its Applications 68, (1996), 133-151. MR 97c:57025

24. C. Murolo, Semidifférentiabilité, transversalité et homologie de stratifications régulières, thesis, University of Provence, 1997.

25. C. Murolo and D. Trotman, Semidifferentiable stratified morphisms, C. R. Acad. Sci. Paris, t 329, Série I, p. 147-152, 1999. MR 2001i:58008

26. C. Murolo and D. Trotman, Relèvements continus de champs de vecteurs, Bull. Sci. Math., 125, 4 (2001), 253-278. MR 2003c:58003

27. H. Natsume, The realisation of abstract stratified sets, Kodai Math. J. 3, (1980), 1-7. MR 81c:57029

28. L. Noirel, Plongements sous-analytiques d'espaces stratifiés de Thom-Mather, thesis, University of Provence, 1996.

29. A. Parusiński, Lipschitz stratifications, Global Analysis in Modern Mathematics (K. Uhlenbeck, ed.), Proceedings of a Symposium in Honor of Richard Palais' Sixtieth Birthday, Publish or Perish, Houston, 1993, 73-91. MR 95e:32008

30. A. A. du Plessis, Continuous controlled vector fields, Singularity theory (Liverpool, 1996, edited by J. W. Bruce and D. M. Q. Mond), London Math. Soc. Lecture Notes 263, Cambridge Univ. Press, Cambridge, (1999), 189-197. MR 2001i:58090

31. M. Teufel, Abstract stratified sets are (b)-regular, Journal of Differential Geometry 16 (1981), 529-536. MR 84f:58010

32. R. Thom, Ensembles et morphismes stratifiés, Bull.A.M.S. 75 (1969), 240-284. MR 39:970

33. D. J. A. Trotman, Geometric versions of Whitney regularity, Annales Scientifiques de l'Ecole Normale Supérieure, 4 ème série, t. 12, (1979), 453-463. MR 81g:58002a 
34. J.-L. Verdier, Stratifications de Whitney et théorème de Bertini-Sard, Inventiones Math. 36 (1976), 295-312. MR 58:1242

35. A. Verona, Stratified mappings - structure and triangulability, Lecture Notes in Math. 1102, Springer-Verlag, Berlin, 1984. MR 86k:58010

36. H. Whitney, Local properties of analytic varieties, Differential and Combinatorial Topology, Princeton Univ. Press, (1965), 205-244. MR 32:5924

Laboratoire d'Analyse, Topologie et Probabilités, Centre de Mathématiques et Informatique, Université de Provence, 39, rue Joliot-Curie, 13453 Marseille Cedex 13, FRANCE

E-mail address: murolo@gyptis.univ-mrs.fr

Laboratoire d'Analyse, Topologie et Probabilités, Centre de Mathématiques et Informatique, Université de Provence, 39, rue Joliot-Curie, 13453 Marseille Cedex 13, FRANCE

E-mail address: trotman@gyptis.univ-mrs.fr

Matematisk Institut, Ny Munkegade, Universitet Aarhus, Aarhus, Denmark

E-mail address: matadp@mi.aau.dk 